\title{
Non-contact multi-spectral imaging combined with thermography to determine physiological changes in perfusion during clinical interventions
}

\author{
by John H. G. M. Klaessens ${ }^{\star}$, Herke Jan Noordmans", Martin W. Nelisse*, Rudolf M. Verdaasdonk ${ }^{*}$ \\ *Department of Medical Technology and Clinical Physics, University Medical Center Utrecht, Utrecht, \\ The Netherlands.J.h.g.m.klaessens@umcutrecht.nl \\ ** Department Physics and Medical Technology, Free University Medical Center, Amsterdam The Netherlands.
}

\begin{abstract}
Reflectance spectroscopy and thermography are used as non- invasive, non-contact imaging method to study the physiology of skin processes or pathophysiology of skin diseases. In this proceeding the development of a multispectral imaging system based on a tunable LED light source is described. The system is validated under laboratory conditions on volunteers. Different algorithms to calculate the $\mathrm{O}_{2} \mathrm{Hb}$ and $\mathrm{HHb}$ changes in the tissue are being developed and the results are presented for different sets of selected wavelengths. Simultaneously thermal images were recorded to image the temperature changes caused by perfusion changes. The first clinical studies have been started.
\end{abstract}

\section{Introduction}

Both reflectance spectroscopy and thermography can be used as a non- invasive, non-contact imaging method to study the physiology of skin processes or pathophysiology of skin diseases. Near infrared (NIR) light has been used for more than 3 decades to study blood and tissue oxygenation changes in animal studies, clinical intervention studies and in oxygen supply and consumption in the brain and muscles [2;5;9;10;12]. Thermography for measuring absolute surface temperatures is recently rediscovered because the new generation thermal cameras have become more practical in use and they are easy to calibrate. Both these techniques can be used to study localized differences in tissue characteristics (e.g. tumor) and dynamic changes in the tissue (e.g. perfusion, oxygenation and temperature changes). In this paper the response of the skin after provocation is described by monitoring changes in temperature and oxygenation induced by tissue perfusion changes. A LED Multi-spectral imaging system has been developed and has been validated. Algorithms are being developed [11] to calculate oxygenation changes in the superficial skin or tissue layer ( $1 \mathrm{~mm}$ depth). In this paper, reflective spectroscopy and thermography are applied to studying the oxygenation and temperature changes in the skin. First proof of principle is shown in laboratory experiments, hereafter the imaging methods are being applied in clinical studies.

\section{Physiology of tissue perfusion}

The microcirculation is defined as that part of the vascular tree with vessels smaller than $100 \mu$ m: arterioles, capillaries and venules. All those vessels expand to many small branches for optimal oxygen exchange to the surrounding tissue cells. The delivery of oxygen to the tissue depends on the red blood cell content en flow (regulation) in the microcirculation[8;13]. The flow can be regulated by variation in arteriolar resistance (vascular smooth muscles) and the number of open and closed capillaries. In muscles and tissue a large number of capillaries are closed due to contraction of the pre-capillary sphincter, this is a reserve capacity that can be opened when local conditions change (e.g. fall in pO2) and extra oxygen is needed. Figure 1 shows a schematic drawing of the blood vessels in the human skin and a table with average depths where the blood vessels are located. The skin is a complex multilayered organ which covers the whole body. The skin can be roughly divided into three layers: epidermis, dermis and the hypodermis. The epidermal, dermis and hypodermis thickness depends on factors such as gender, age, skin type, and anatomic position[7]. The most superficial blood vessels are located in the dermis at a depth less than $2 \mathrm{~mm}$. The skin is an exceptional organ were the blood supply even under normal conditions can vary extremely. This is caused by the thermo regulating function of the skin. Several physiological and patho-physiological processes (aging, smoking, cancer wounds) may influence the blood flow to the skin and consequently influence the partial oxygen pressure (pO2) in the skin[14]. Healthy skin has a high pO2 level $(8-13 \mathrm{kPa}$ ) but in tumors or hypoxic wounds the pO2 level can fall to $0.7 \mathrm{kPa}[6 ; 15]$. Another process that can influence the skin pO2 is anesthesia, different type of anesthetics influence the skin perfusion in different ways[1]. 


\begin{tabular}{|c|c|c|c|}
\hline \multicolumn{2}{|c|}{ Skin } & Thickness & $\begin{array}{l}\text { Blood content } \\
\text { norman/enthema }\end{array}$ \\
\hline Epidermis & Stratum corneum & $100 \mu \mathrm{m}$ & $0 / 0 \%$ \\
\hline \multirow{4}{*}{ Dermis } & Papillary dermis & $160 \mu \mathrm{m}$ & $2 / 8 \%$ \\
\hline & Upper blood plexus & $80 \mu \mathrm{m}$ & $5 / 20 \%$ \\
\hline & Reticular dermis & $1500 \mu \mathrm{m}$ & $1 / 4 \%$ \\
\hline & Lower blood plexus & $200 \mu \mathrm{m}$ & $5 / 20 \%$ \\
\hline Hypodermis & & $\sim 2000 \mu \mathrm{m}$ & \\
\hline
\end{tabular}

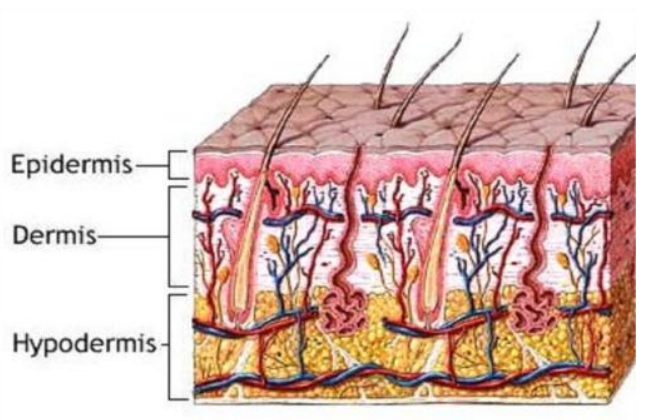

Fig. 1 Schematic drawing of the skin with its blood vessels. The average depth of the back scattered light is maximal around $2 \mathrm{~mm}$ this is deep enough to reach the dermis levels that contain blood.

\section{Material and Methods}

Recently high power, low cost LEDs have become available in different wavelengths ranges and with bandwidths of approximately $30-80 \mathrm{~nm}$. This gives the possibility to build flashing and continuous light sources from the UV to the near infrared light wavelength ranges with the necessary power for different research questions. In our department a tunable VIS-NIR LED light source $(370 \mathrm{~nm}-880 \mathrm{~nm})$ has been developed. A 2-D flat panel with a total of 600 LEDs, consisting of 17 different wavelengths, a CCD camera is mounted in the middle of the panel (figure 2).
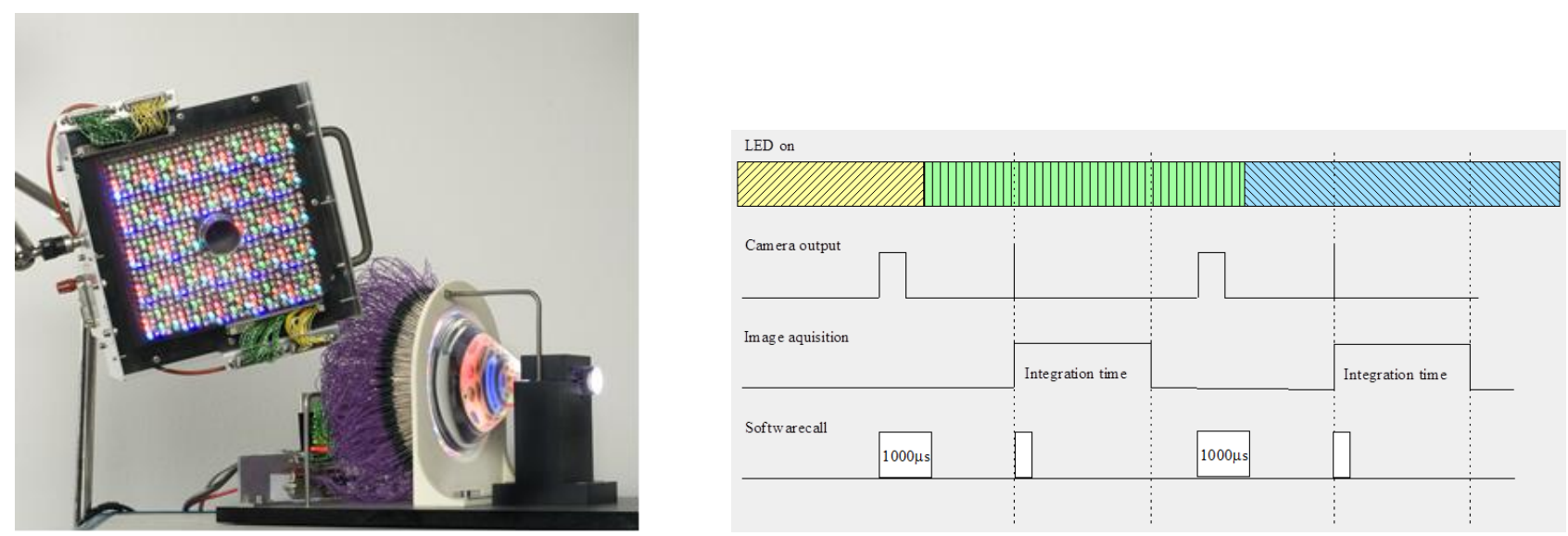

Fig 2 The tunable LED light source for the multi-spectral imaging system. On the right the scheme of controlling the switching of the LEDs and the integration time of the camera.

The LED controllers are tuned that all the wavelengths give the same intensity and the LEDs are arranged over the panel so the light is homogeneous distributed over the subject. The camera is synchronized with the flashing sequence of the LEDs. The software controls which LEDs will be turned on and for how long. The first software call will give an output trigger for the camera that will turn on the specified LEDs, the second software call will start the integration of the camera for a specified time. This guaranties that the collected light is always in the stable period of the LEDs and that only the intended LED light is collected (no overlap of wavelengths).

The LEDs can be flashed in high speed, the limitation is the throughput rate of the camera. But for studying most physiological phenomena $10 \mathrm{~Hz}$ is fast enough.

Simultaneously with the multispectral imaging the hand is imaged with a Thermal camera. Thermography was performed with a calibrated IR thermal camera (FLIR ThermoCam SC640, Seattle, USA), uncooled microbolometer with a $640 \times 480$ pixel array. The sensor has 14 bit dynamic range and the sensitivity is from 7.5 to $13.5 \mu \mathrm{m}$. The data were recorded and analyzed with the ThermaCamTM Researcher 2007 Pro 2.9 (FLIR systems AB, Sweden) software 
coupled by an IEEE-1394 FireWire interface with a laptop PC. The thermal camera produces a matrix of temperature values; each pixel in the thermographic image represents one temperature. The data were collected with a temperature resolution of 0.1 degree Kelvin $(\mathrm{K})$ and the thermal images were acquired with a 1 second interval. Offline the data were analyzed with software written in MatLab. The LED light used for the multi-spectral imaging did not heat the skin surface. The thermal camera was at a distance of 1 meter from the hand. The emissivity of the skin was set to 0.99.

The measuring systems and analyzing methods were tested under ideal conditions (dark) and in hospital situation (ambient light). And temperature and oxygenation changes were induced during arm clamping experiments on volunteers (Figure 3). The arm is clamped using a blood pressure cuff attached to the upper arm. A pressure of 240 $\mathrm{mmHg}$ was applied for 5 minutes to block all the blood flow to the arm. Images at the normoxic, hypoxic and reperfusion stage with the two systems are collected and analyzed and the results will be presented.

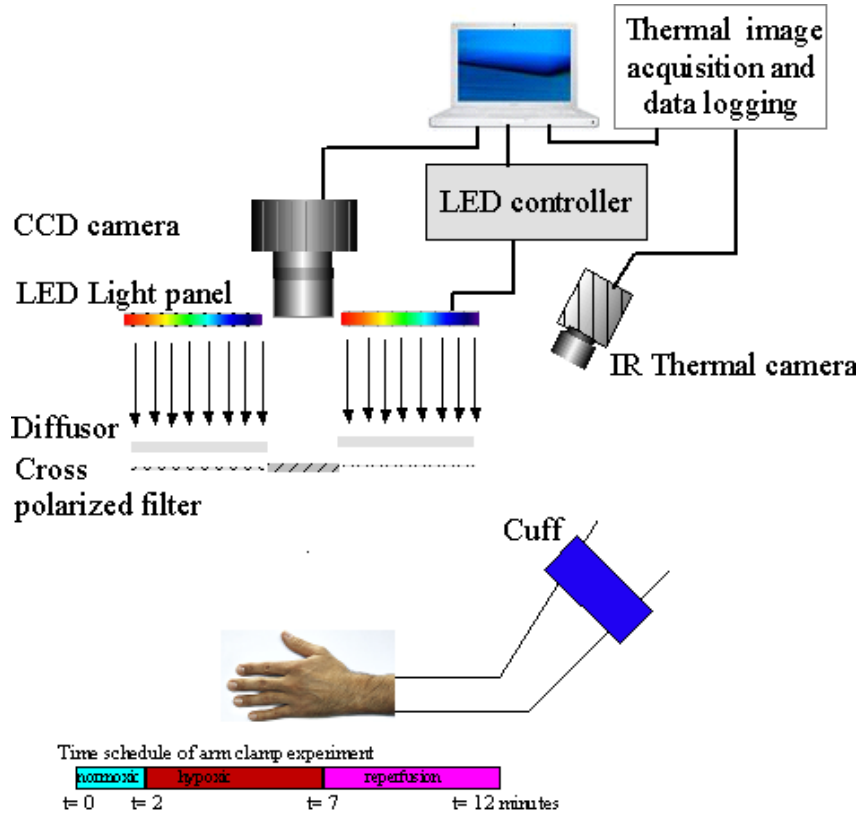

Physiological response to arterial and venous occlusion:

1 Blood flow stops:

$\rightarrow$ Constant $\mathrm{tHb}$

$\rightarrow$ Temperature decreases

2 Consumption of oxygen:

$\rightarrow$ Increase $\mathrm{HHb}$

$\rightarrow$ Decrease $\mathrm{O}_{2} \mathrm{Hb}$

3 The temperature in tissue cannot be controlled by blood circulation:

$\rightarrow$ Decrease temperature

perfusion:

1 Hyperperfusion

$\rightarrow$ Increase $\mathrm{O}_{2} \mathrm{Hb}$

$\rightarrow$ Decrease $\mathrm{HHb}$

2 Increase in temperature

Fig. 3: The experimental setup of the arm clamp experiment: the LED illumination around the CCD camera. A selected set of LEDs flash, the light is diffused homogeneously over the object (hand) and the backscattered light from the tissue is received by the CCD camera. The time schedule of the clamping is 2 minutes normoxic skin, 5 minutes clamping with total occlusion, 5 minutes registration after reperfusion. The thermographic images were acquired with a 1 second interval.

\section{Algorithm for skin oxygenation}

The backscattered light was analyzed by calculating the $\mathrm{O}_{2} \mathrm{Hb}$ and $\mathrm{HHb}$ concentration changes. Three different algorithms were applied: delta time method ( $\mathrm{dt}$ or $\Delta \mathrm{t}$ ), the delta wavelength method ( $\mathrm{dl}$ or $\Delta \lambda$ ) and the Fit method (FIT). In short these methods are described.

The attenuation of light in tissue is caused by absorption and scattering. The absorption can be divided in two parts: one due to chromophores with constant tissue concentration and one due to chromophores with a variable concentration over time. In skin tissue, the main varying components are the concentrations of oxy- and deoxyhemoglobin $\left(\mathrm{O}_{2} \mathrm{Hb}\right.$ and $\left.\mathrm{HHb}\right)$. These concentrations depend on the oxygen saturation and the total blood volume in the tissue. The absorbance $A$ in optical densities (OD) is defined as:

$$
A=-{ }^{10} \log \left(\frac{I}{I_{0}}\right)=-{ }^{10} \log T={ }_{n} \Sigma \varepsilon_{n} c_{n} d
$$

$I_{0}$ is the intensity of the incident light, $I$ the intensity of the received light, $T$ is the transmission $I / I_{0}, d$ is the optical path-length in the tissue, $\varepsilon$ is the molar extinction coefficient $\left[\mathrm{M}^{-1} \mathrm{~cm}^{-1}\right]$ describing the absorption per molar concentration of the substance per centimeter of optical path, $c$ the concentration of the substance [M] summed over all the particle types $(n)$. Scattering causes light propagation in all directions, which increases the light path through the tissue and thus influences the received light intensity. All together these effects can be comprised in the modified Lambert-Beer law[3] (MLBL):

$$
\bar{A}(\lambda)=\overline{\bar{\varepsilon}}(\lambda) \bar{c}(t) \overline{D P F}(\lambda) d+\bar{G}(\lambda)+\bar{H}(t)
$$


With $\bar{A}(\lambda)$ the measured absorbance $[-], \quad \bar{\varepsilon}(\lambda)$ the molar extinction coefficients $\left[\mathrm{mM}^{-1} \mathrm{~cm}^{-1}\right], \quad \bar{c}(\mathrm{t})$ the concentration of molecules [mM], $\bar{G}(\lambda)$ the oxygen independent and wavelength dependent losses [-], $\bar{H}(t)$ are the oxygen independent and time dependent losses [-], $d$ is the source detector distance [cm], $\overline{D P F}(\lambda)$ is the differential path length factor [-]. $\operatorname{DPF}(\lambda)$ is the differential path-length factor, which corrects the geometrical source-detector distance to the mean optical path in the tissue. $G(\lambda)$ and $H(t)$ are the oxygen independent loss caused by scattering, absorption, geometry and other boundary losses[2;4].

Equation 3-2 can be written out for multiple wavelengths to calculate the concentration of chromophores in the skin. Equation 3-2 can for example be written out for two chromophores $\mathrm{O}_{2} \mathrm{Hb}$ and $\mathrm{HHb}$ and 3 wavelengths:

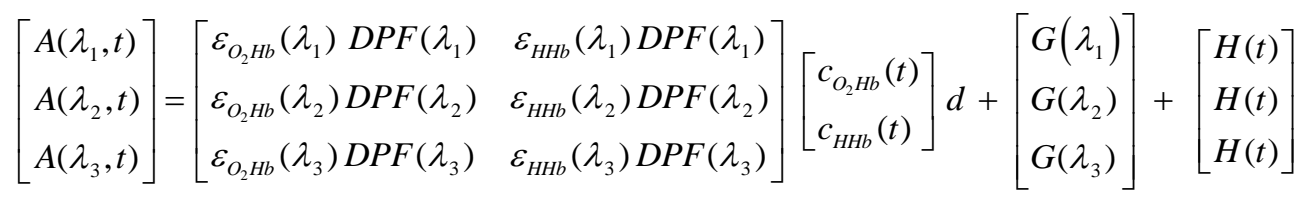

Two different methods can be applied to calculate the concentration changes; the classical $\Delta \mathrm{t}-\mathrm{method}$ and the $\Delta \lambda$-method[11]. In the classical $\Delta t$-method the assumption is made that the $H$ term is zero, or constant in time. Calculating the difference in concentrations over a time period $(\Delta t)$ relative to a stable starting point the absolute concentration changes can be calculated using known DPF values of the interrogated tissue from literature. This leads to the formula for the chromophore concentrations:

$$
\Delta_{t} \bar{c}=\frac{\overline{\overline{\varepsilon D P F}}^{-1} \Delta_{t} \bar{A}}{d}
$$

The $\Delta \lambda$-method is derived from equation 4.3 under the assumptions; the optical path-length is wavelength dependent but constant in time during the experiments, the geometry factor $G$ is wavelength dependent and the geometry factor $H$ is time dependent. The equation 4.3 is written out as 3 equations using 3 absorption wavelengths:

$$
\begin{aligned}
& A\left(\lambda_{1}, t\right)=\varepsilon\left(\lambda_{1}\right)_{O_{2} H b} c_{O_{2} H b}(t) D P F\left(\lambda_{1}\right) d+\varepsilon\left(\lambda_{1}\right)_{H H b} c_{H H b}(t) D P F\left(\lambda_{1}\right) d+G\left(\lambda_{1}\right)+H(t) \\
& A\left(\lambda_{2}, t\right)=\varepsilon\left(\lambda_{2}\right)_{O_{2} H b} c_{O_{2} H b}(t) \operatorname{DPF}\left(\lambda_{2}\right) d+\varepsilon\left(\lambda_{2}\right)_{H H b} c_{H H b}(t) \operatorname{DPF}\left(\lambda_{2}\right) d+G\left(\lambda_{2}\right)+H(t) \\
& A\left(\lambda_{3}, t\right)=\varepsilon\left(\lambda_{3}\right)_{O_{2} H b} \quad c_{O_{2} H b}(t) D P F\left(\lambda_{3}\right) d+\varepsilon\left(\lambda_{3}\right)_{H H b} c_{H H b}(t) D P F\left(\lambda_{3}\right) d+G\left(\lambda_{3}\right)+H(t)
\end{aligned}
$$

Solving the equations by taking the differences over the wavelengths leads to elimination of the time dependent and wavelength independent geometry factor $\mathrm{H}(\mathrm{t})$. Assuming that the $G$ terms are wavelength independent, is not realistic and the $G$ terms cannot be neglected they have to be eliminated from the formula by applying the delta time method. This gives for the concentration changes for the $\Delta \lambda$-method:

$$
\begin{aligned}
& \left(\varepsilon\left(\lambda_{1}\right)_{H H b} D P F\left(\lambda_{1}\right)-\varepsilon\left(\lambda_{3}\right)_{H H b} D P F\left(\lambda_{3}\right)\right)\left[\Delta_{t \lambda 12} A(t)\right]- \\
& \Delta_{t} c_{O_{2} H b}(t)=\frac{\left(\varepsilon\left(\lambda_{1}\right)_{H H b} D P F\left(\lambda_{1}\right)-\varepsilon\left(\lambda_{2}\right)_{H H b} D P F\left(\lambda_{2}\right)\right)\left[\Delta_{t} \lambda_{13} A(t)\right]}{d\left[\left(\varepsilon\left(\lambda_{1}\right)_{H H b} D P F\left(\lambda_{1}\right)-\varepsilon\left(\lambda_{3}\right)_{H H b} D P F\left(\lambda_{3}\right)\right)\left(\varepsilon\left(\lambda_{1}\right)_{O_{2} H b} D P F\left(\lambda_{1}\right)-\varepsilon\left(\lambda_{2}\right)_{O_{2} H b} D P F\left(\lambda_{2}\right)\right)-\right.} \\
& \left.\left(\varepsilon\left(\lambda_{1}\right)_{H H b} \operatorname{DPF}\left(\lambda_{1}\right)-\varepsilon\left(\lambda_{2}\right)_{H H b} \operatorname{DPF}\left(\lambda_{2}\right)\right)\left(\varepsilon\left(\lambda_{1}\right)_{O_{2} H b} D P F\left(\lambda_{1}\right)-\varepsilon\left(\lambda_{3}\right)_{O_{2} H b} D P F\left(\lambda_{3}\right)\right)\right] \\
& \left(\varepsilon\left(\lambda_{1}\right)_{O_{2} H b} D P F\left(\lambda_{1}\right)-\varepsilon\left(\lambda_{3}\right)_{O_{2} H b} D P F\left(\lambda_{3}\right)\right) \Delta_{t \lambda 12} A(t)- \\
& \Delta_{t} c_{H H b}(t)=\frac{\left(\varepsilon\left(\lambda_{1}\right)_{O_{2} H b} D P F\left(\lambda_{1}\right)-\varepsilon\left(\lambda_{2}\right)_{O_{2} H b} D P F\left(\lambda_{2}\right)\right) \Delta_{t \lambda 13} A(t)}{d\left[\left(\varepsilon\left(\lambda_{1}\right)_{O_{2} H b} D P F\left(\lambda_{1}\right)-\varepsilon\left(\lambda_{3}\right)_{O_{2} H b} D P F\left(\lambda_{3}\right)\right)\left(\varepsilon\left(\lambda_{1}\right)_{H H b} D P F\left(\lambda_{1}\right)-\varepsilon\left(\lambda_{2}\right)_{H H b} D P F\left(\lambda_{2}\right)\right)-\right.} \\
& \left.\left(\varepsilon\left(\lambda_{1}\right)_{O_{2} H b} D P F\left(\lambda_{1}\right)-\varepsilon\left(\lambda_{2}\right)_{O_{2} H b} D P F\left(\lambda_{2}\right)\right)\left(\varepsilon\left(\lambda_{1}\right)_{H H b} D P F\left(\lambda_{1}\right)-\varepsilon\left(\lambda_{3}\right)_{H H b} D P F\left(\lambda_{3}\right)\right)\right]
\end{aligned}
$$

The delta FIT method uses the pseudo inverse method Moore-Penrose to match the found spectra with the literature reflection spectra and is a generalization of the delta wavelength method. This method can be used with more than three wavelength

In situation were consumption of oxygen stays constant and the total blood volume changes because of vasodilatation then the total blood volume $(\mathrm{tHb})$ is changing:

$$
\Delta \mathrm{Hbt}=\Delta \mathrm{cO} \mathrm{O}_{2} \mathrm{Hb}+\Delta \mathrm{cHHb}
$$

The collected multi spectral images of the skin surface can be converted to the reflectance spectrum by correcting them for spatially non uniform light distribution and the camera dark current:

$$
R(t, \lambda)=\frac{S(t, \lambda)-D(\lambda)}{W_{\mathrm{Ref}}(t, \lambda)-D(\lambda)}
$$


With $S(\lambda)$ the measured light CCD camera, $D(\lambda)$ the dark current signal and $W_{\text {ref }}(\lambda)$ the reference of $100 \%$ reflectance of a white standard. By replacing the $A$ by the $R$ oxygen changes during interventions can be calculated in the backscatter mode.

\subsection{Ambient light correction}

The ambient light In the operating room is not constant in intensity (movement of surgery lightning, movement of persons) and it is not always possible to dim the light in the room. These fluctuations in ambient light will superimposed on each of the collected wavelength images of the multispectral imaging system.

To calculate the oxygenation changes in tissue the tunable LED light source will flash sequentially 3 or more wavelengths. For the validation experiments was chosen to use 7 wavelengths in the visible and near infrared region. The wavelengths were selected based on the absorption spectra of oxy and deoxy-hemoglobin, the spectra must be different for the selected wavelengths.

We selected the sequence: 470, 525, 625, 690. 750, 810 and $850 \mathrm{~nm}$. These wavelengths were repeatedly flashed during the period of the experiment (12 minutes). The time between the wavelengths was 10 milliseconds and the time between the sequences was set to $800 \mathrm{~ms}$ this resulted in one sequence per second.

To correct for the fluctuations in the ambient light an extra image was acquired during each sequence with the LED lights turned off. This acquired image was subtracted from the other wavelength images in that sequence.

\section{Results}

\subsection{Laboratory measurement: total occlusion left arm}

One example of the results of the arm clamping experiment described in figure 3 is shown below (figure 4).
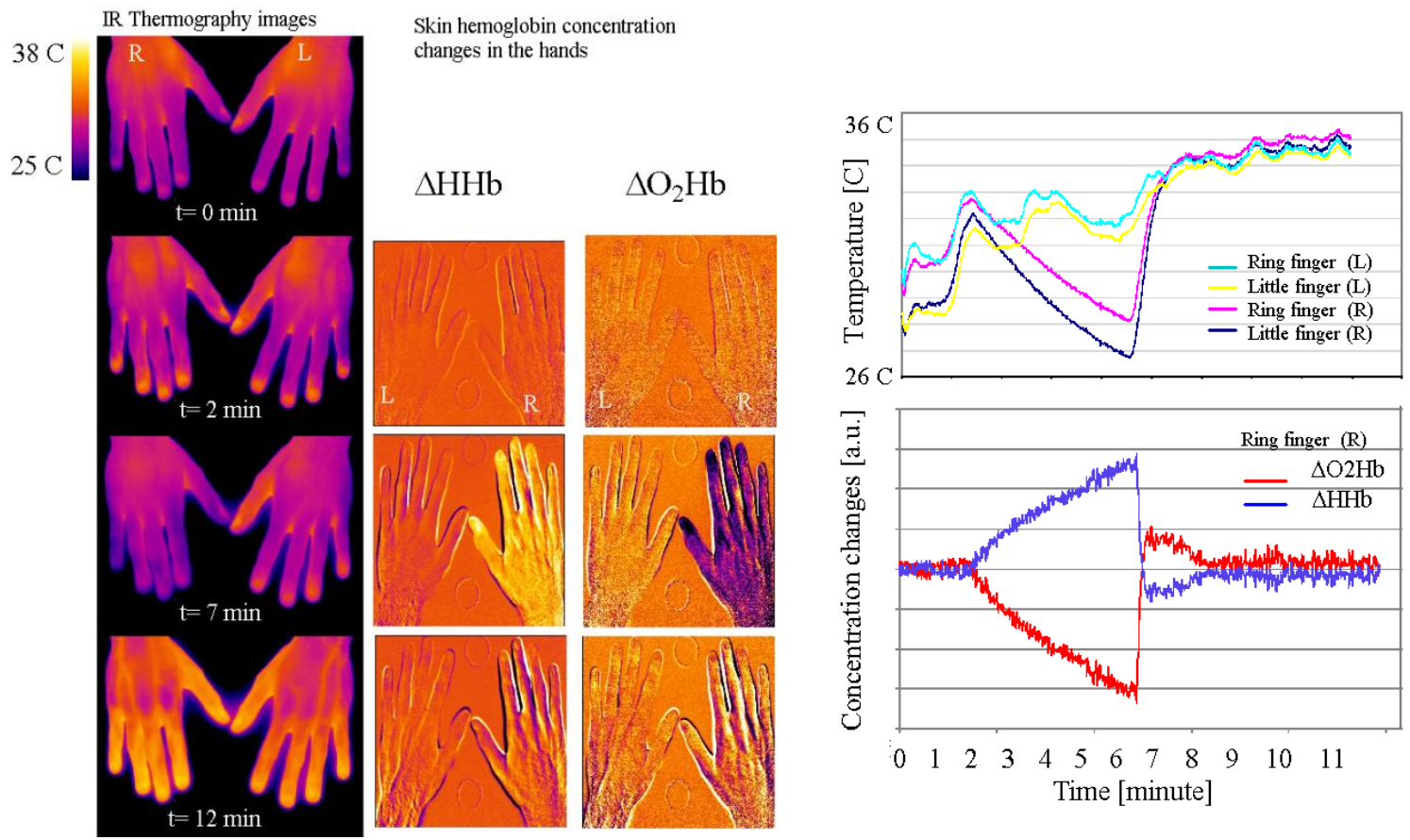

Fig. 4 The total occlusion is performed on the right arm. On the left the images show a cooling of the hand and a large change in oxygenation $\left(\mathrm{O}_{2} \mathrm{Hb}\right.$ and $\left.\mathrm{HHb}\right)$ till $t=7 \mathrm{~min}$, thereafter the temperature increases and the oxygenation changes opposite way. On the right the graphs of the temperature changes and the oxygenation changes, during the whole experiment, in a region of interest (finger tip right hand) are presented.

The measured temperature and the oxygenation changes in the hands show the expected changes as described in figure 3. The temperature and the oxygenation changes in the hand were analysed in regions of interest on the fingers and as images of the total hand (figure 4). 
Correction algorithms for the influence of ambient light were tested successfully during controlled light fluctuations in our laboratory.

The first results show that the delta wavelength method is less sensitive for movement artifacts.

Both measuring systems are momentary used in several clinical studies to measure local oxygenation changes and temperature changes during: skin flap transplantation (ENT), local block anesthesia for hand surgery and epileptic brain surgery.

\subsection{Influence of wavelengths and concentration algorithm}

The concentration algorithms ( $\Delta \mathrm{t}, \Delta \lambda$ and FIT method) were tested for 7 or 8 different wavelength combinations (fig. 5). For all the arm clamping registrations by volunteers the concentration changes were calculated. An example of the calculated concentration changes for $\Delta$ t method for the 8 wavelengths combinations are shown in Figure 6

\begin{tabular}{|c|c|c|c|c|c|c|c|}
\hline $\mathrm{d} \Lambda$ & 1 & 2 & 3 & 4 & 5 & 6 & 7 \\
\hline 470 & - & - & - & - & - & - & - \\
\hline 525 & - & - & - & - & $\mathrm{X}$ & $\mathrm{X}$ & $\mathrm{X}$ \\
\hline 625 & - & - & - & - & - & - & - \\
\hline 690 & - & $\mathrm{X}$ & $\mathrm{X}$ & $\mathrm{X}$ & - & $\mathrm{X}$ & $\mathrm{X}$ \\
\hline 750 & $\mathrm{X}$ & $\mathrm{X}$ & - & $\mathrm{X}$ & $\mathrm{X}$ & $\mathrm{X}$ & - \\
\hline 810 & $\mathrm{X}$ & - & $\mathrm{X}$ & $\mathrm{X}$ & - & - & - \\
\hline 850 & $\mathrm{X}$ & $\mathrm{X}$ & $\mathrm{X}$ & - & $\mathrm{X}$ & - & $\mathrm{X}$ \\
\hline
\end{tabular}

\begin{tabular}{|c|c|c|c|c|c|c|c|c|}
\hline dt FIT & 1 & 2 & 3 & 4 & 5 & 6 & 7 & 8 \\
\hline 470 & - & - & - & - & - & - & - & - \\
\hline 525 & $\mathrm{X}$ & - & $\mathrm{X}$ & $\mathrm{X}$ & $\mathrm{X}$ & $\mathrm{X}$ & - & - \\
\hline 625 & $\mathrm{X}$ & $\mathrm{X}$ & $\mathrm{X}$ & - & - & - & - & - \\
\hline 690 & $\mathrm{X}$ & $\mathrm{X}$ & - & - & - & $\mathrm{X}$ & $\mathrm{X}$ & $\mathrm{X}$ \\
\hline 750 & $\mathrm{X}$ & $\mathrm{X}$ & $\mathrm{X}$ & $\mathrm{X}$ & $\mathrm{X}$ & $\mathrm{X}$ & $\mathrm{X}$ & $\mathrm{X}$ \\
\hline 810 & $\mathrm{X}$ & $\mathrm{X}$ & - & - & $\mathrm{X}$ & $\mathrm{X}$ & $\mathrm{X}$ & - \\
\hline 850 & $\mathrm{X}$ & $\mathrm{X}$ & $\mathrm{X}$ & $\mathrm{X}$ & $\mathrm{X}$ & $\mathrm{X}$ & $\mathrm{X}$ & $\mathrm{X}$ \\
\hline
\end{tabular}

Fig. 5 The wavelengths choices for the three different concentration algorithms.

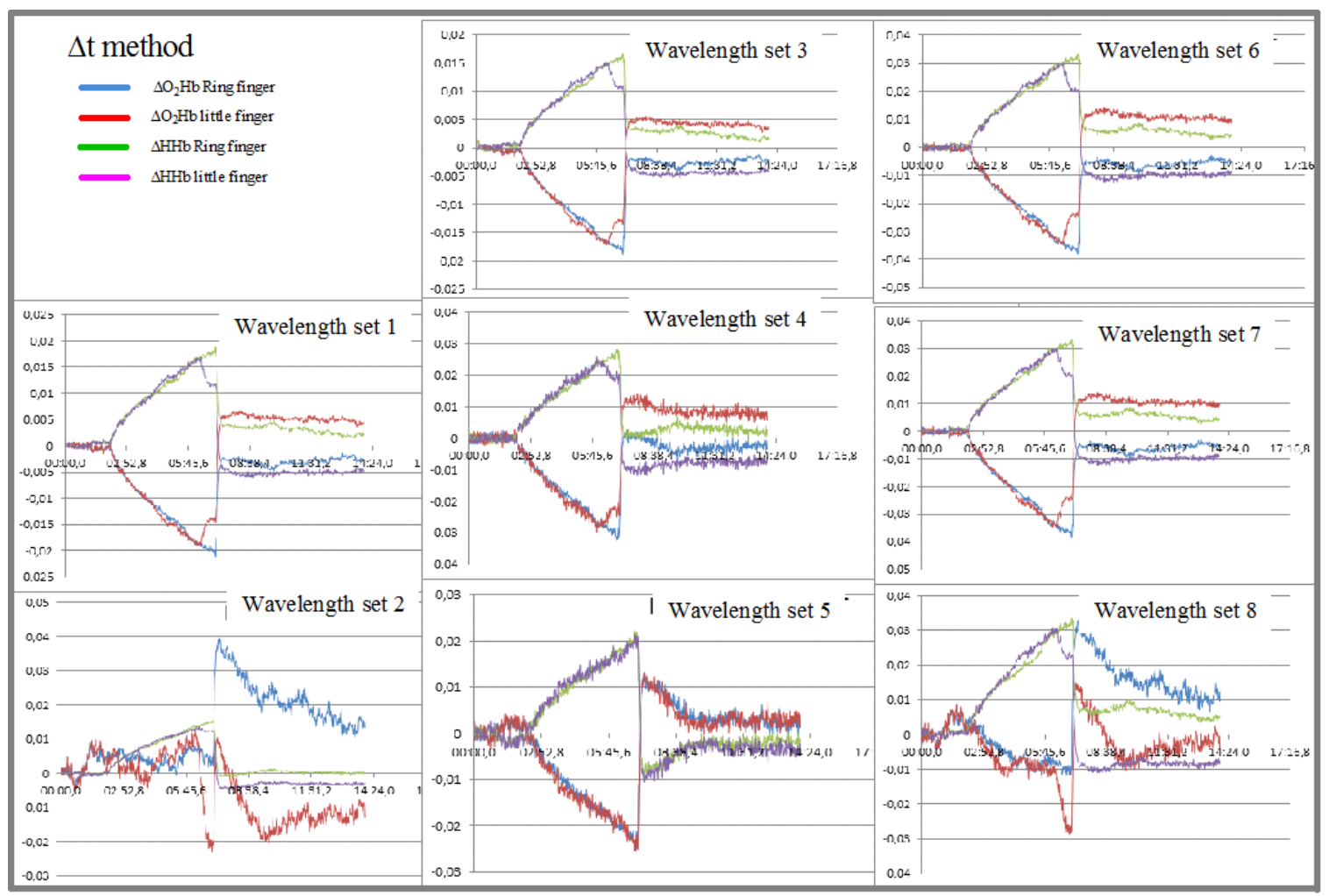

Fig. 6 An example of the concentration changes by one volunteer during arm clamping, using the $\Delta t$ method and all the 8 wavelength sets.

The results in figure 6 show correct and disturbed (not correct) concentration changes according to the expected concentration changes. Also the noise levels are influenced by the method and the choice of wavelengths. To qualify the concentration changes all the calculated concentration changes (for all volunteers, for all methods and for all 
wavelengths sets) were scored (2 correct, 1 partially correct and 0 wrong). This was also done for the noise levels (2 low noise levels, 1 some noise and 0 large noise levels). The results are presented in table 1.

\begin{tabular}{|c|c|c|c|c|c|c|}
\hline \multirow{2}{*}{$\begin{array}{l}\text { method } \\
\text { wavelength }\end{array}$} & \multicolumn{2}{|l|}{$\Delta \mathrm{t}$} & \multicolumn{2}{|l|}{ FIT } & $\Delta \lambda$ & \multirow[b]{2}{*}{$\frac{n}{2}$} \\
\hline & Signal & $\frac{n}{0}$ & Signal & $\frac{n}{0}$ & Signal & \\
\hline 1 & 6 & 1 & 3 & 0 & 3 & 0 \\
\hline 2 & 2 & 6 & 3 & 0 & 0 & 0 \\
\hline 3 & 6 & 6 & 6 & 0 & 3 & 0 \\
\hline 4 & 6 & 5 & 6 & 6 & 2 & 0 \\
\hline 5 & 6 & 3 & 6 & 0 & 6 & 5 \\
\hline 6 & 6 & 6 & 6 & 1 & 6 & 6 \\
\hline 7 & 3 & 5 & 0 & 0 & 6 & 6 \\
\hline 8 & 4 & 3 & 1 & 0 & & \\
\hline
\end{tabular}

Table 1 The quality of the methods for the different wavelength sets.

The higher the value the better the signal or noise is. The yellow combinations give good results,

The concentration algorithms give for all three methods good results when using the following wavelengths: $\Delta t$ method with $525 \mathrm{~nm}, 625 \mathrm{~nm}$ and $850 \mathrm{~nm}, \Delta \lambda$ method whit $525 \mathrm{~nm}, 750 \mathrm{~nm}$ and $850 \mathrm{~nm}$, FIT method $525 \mathrm{~nm}$, 690nm and $750 \mathrm{~nm}$ (or 850nm).

\section{Conclusion}

The results of the validation study of the LED based multi-spectral imaging system show clearly that this system can measure the perfusion and oxygenation changes caused by physiological changes in the tissue during arm clamping and this is successfully confirmed and correlated with absolute temperature measurements of the thermal camera.

The concentration algorithms give for all three methods good results if the correct wavelength are used, more clinical studies need to be done to optimize the algorithm for specific medical applications.

The first results show that the delta wavelength method is less sensitive for movement artifacts and could been used for real-time oxygenation imaging in moving objects.

Clinical studies are ongoing during at this moment: skin flap transplantations (ENT), regional anesthetic blocks for hand surgery, open epileptic brain surgery (localisation of epileptic center), the first results look promising.

Thermography and multi-spectral imaging prove to be a promising techniques for real-time diagnostics of physiological processing in medicine.

[1] Z. Abramovic, M. Sentjurc, J. Kristl, N. Khan, H. Hou, H.M. Swartz, Influence of different anesthetics on skin oxygenation studied by electron paramagnetic resonance in vivo. Skin Pharmacol.Physiol vol.20, pp.77-84, 2007.

[2] D.T. Delpy, M. Cope, Quantification in tissue near-infrared spectroscopy. Philosophical Transactions of the Royal Society B-Biological Sciences vol.352, pp.649-659, 1997.

[3] D.T. Delpy, M. Cope, P. van der Zee, S. Arridge, S. Wray, J. Wyatt, Estimation of optical pathlength through tissue from direct time of flight measurement. .Phys.Med.Biol. vol.33, pp.1433-1442, 1988.

[4] A.D. Edwards, C. Richardson, Z.P. van der, C. Elwell, J.S. Wyatt, M. Cope, D.T. Delpy, E.O. Reynolds, Measurement of hemoglobin flow and blood flow by near-infrared spectroscopy. J.Appl.Physiol vol.75, pp.18841889, 1993.

[5] M. Ferrari, L. Mottola, V. Quaresima, Principles, techniques, and limitations of near infrared spectroscopy. Can.J.Appl.Physiol vol.29, pp.463-487, 2004.

[6] B. Gallez, C. Baudelet, B.F. Jordan, Assessment of tumor oxygenation by electron paramagnetic resonance: principles and applications. NMR Biomed. vol.17, pp.240-262, 2004.

[7] T. Gambichler, A. Orlikov, R. Vasa, G. Moussa, K. Hoffmann, M. Stucker, P. Altmeyer, F.G. Bechara, In vivo optical coherence tomography of basal cell carcinoma. J.Dermatol.Sci. vol.45, pp.167-173, 2007.

[8] M. Intaglietta, P.C. Johnson, R.M. Winslow, Microvascular and tissue oxygen distribution. Cardiovasc.Res. vol.32, pp.632-643, 1996.

[9] F.F. Jobsis, Noninvasive, infrared monitoring of cerebral and myocardial oxygen sufficiency and circulatory parameters. Science vol.198, pp.1264-1267, 1977. 
[10] J.H. Klaessens, J.C. Hopman, K.D. Liem, S.H. van Os, J.M. Thijssen, Effects of skin on bias and reproducibility of near-infrared spectroscopy measurement of cerebral oxygenation changes in porcine brain. J.Biomed.Opt. vol.10, pp.44003, 2005.

[11] J.H.G.M. Klaessens, H.J. Noordmans, R. de Roode, R.M. Verdaasdonk, Non-invasive skin oxygenation imaging using a multi-spectral camera system: effectiveness of various concentration algorithms applied on human skin. SPIE Vol: 7174, 2009.

[12] P.L. Madsen, N.H. Secher, Near-infrared oximetry of the brain. Prog.Neurobiol. vol.58, pp.541-560, 1999.

[13] R.N. Pittman, Oxygen transport and exchange in the microcirculation. Microcirculation. vol.12, pp.59-70, 2005.

[14] J. Ratnaraj, B. Kabon, M.R. Talcott, D.I. Sessler, A. Kurz, Supplemental oxygen and carbon dioxide each increase subcutaneous and intestinal intramural oxygenation. Anesth.Analg. vol.99, pp.207-211, 2004.

[15] A.A. Tandara, T.A. Mustoe, Oxygen in wound healing--more than a nutrient. World J.Surg. vol.28, pp.294-300, 2004. 